\title{
Three-dimensional display of a horizontal-parallax-only hologram
}

\author{
You Seok Kim, ${ }^{1}$ Taegeun Kim, ${ }^{1, *}$ Ting-Chung Poon, ${ }^{2}$ and Jin Tae Kim ${ }^{3}$ \\ 'Department of Optical Engineering, Sejong University, 98 Gunja-dong, Gwangjin-gu, Seoul 134-747, South Korea \\ ${ }^{2}$ Department of Electrical and Computer Engineering, Virginia Tech, Blacksburg, Virginia 24061, USA \\ ${ }^{3}$ Department of Photonic Engineering, Chosun University, 375 Seosuk-dong, Dong-gu, Gwangju 501-759, South Korea \\ *Corresponding author: takim@sejong.ac.kr
}

Received 23 July 2010; revised 11 November 2010; accepted 12 November 2010; posted 17 November 2010 (Doc. ID 132198); published 7 February 2011

\begin{abstract}
We propose a three-dimensional (3D) holographic display by converting an optically recorded complex full-parallax (FP) hologram to an off-axis horizontal-parallax-only (HPO) hologram. First, we record the complex FP hologram of an object using optical scanning holography. We then convert the complex FP hologram to an off-axis HPO hologram through fringe-matched Gaussian low-pass filtering and with the introduction of an off-axis reference. Finally, we reconstruct the off-axis HPO hologram optically using an amplitude-only spatial light modulator. Until now, only computer-generated HPO holograms have been displayed optically. To the best of our knowledge, this is the first demonstration of a 3D display of an optically recorded HPO hologram. (C) 2011 Optical Society of America
\end{abstract}

OCIS codes: $\quad 090.1995,090.2870$.

\section{Introduction}

Electroholographic systems that reconstruct a computer-generated hologram (CGH) have been proposed for three-dimensional (3D) display in which the CGH has been synthesized as a form of off-axis horizontal-parallax-only (HPO) holographic data [1-5]. This makes it possible to display computer-generated $3 \mathrm{D}$ images without twin-image noise, while reducing the amount of holographic data. However, electroholographic display systems are limited in reconstructing computer-synthesized images of some fictitious $3 \mathrm{D}$ objects because the hologram is generated using computer-aided design (CAD) data. Recently, HPO optical scanning holography (OSH) has been suggested as an electro-optical technique that actually records the holographic information of a real object without vertical parallax [6]. However, the proposed HPO $\mathrm{OSH}$ will introduce aberration upon optical reconstruction along the vertical direction if the vertical extent of the asymmetrical Fresnel zone plate (FZP),

0003-6935/11/070B81-07\$15.00/0

(C) 2011 Optical Society of America which has been proposed to generate the HPO data from a real object, is not small enough [6,7]. To eliminate aberration caused by the asymmetrical FZP, an algorithm that converts a recorded full-parallax (FP) hologram to an HPO hologram was subsequently proposed [7]. In the algorithm, a complex FP hologram is converted to a HPO complex hologram via digital filtering. Digital filtering consists of two parts: Gaussian low-pass filtering (GLF) and fringe-matched (FM) filtering. The purpose of FM filtering is to eliminate the curvature along the vertical direction. Finally, the filtered hologram becomes an encoded pattern between the object's intensity and a one-dimensional (1D) FZP, which does not introduce aberration along the vertical direction upon optical reconstruction regardless of the vertical extent of the 1D FZP. As far as we know, there exists another technique that has shown the recording of holographic data containing the horizontal-parallax effect. The technique synthesizes a modified HPO hologram using multiple viewpoint recordings of an object using a CCD camera [8]. In this paper, we propose to digitally convert the $\mathrm{HPO}$ complex hologram to an off-axis HPO real hologram 
and reconstruct the off-axis HPO real hologram using a conventional amplitude-only spatial light modulator (SLM). This allows us to reconstruct the 3D image of a real object with horizontal parallax but without twin-image noise. To the best of our knowledge, this is the first time the reconstruction of an optically recorded HPO hologram has been demonstrated. Reconstruction of HPO holograms has been, hitherto, using computer-generated data.

In Section 2, we briefly review OSH that can record the complex FP hologram of an object. In Section 3, we review the algorithm that converts the complex FP hologram to an HPO complex hologram [7]. In Section 4 , we propose a digital processing technique that converts the HPO complex hologram to an offaxis HPO complex hologram by adding an off-axis reference. Taking the real part of the resulting HPO complex hologram with added bias becomes the off-axis HPO real hologram. The off-axis HPO real hologram is now a positive real value and can be represented on an amplitude-only SLM. Finally, the reconstruction of the off-axis HPO real hologram using the amplitude-only SLM gives a 3D image without twin-image noise but with horizontal parallax. Finally, in Section 5, the experimental results of the conversion to an off-axis HPO real hologram and $3 \mathrm{D}$ display using the hologram is presented.

\section{Optical Scanning Holography}

$\mathrm{OSH}$ is a form of digital holography that records the complex FP hologram of an object by active optical scanning [9]. The details of OSH shown in Fig. 1 have been intensively investigated previously [10]. In this section, we will briefly review the principles of $\mathrm{OSH}$ and introduce HPO OSH. OSH uses a Mach-Zehnder interferometer and an electronic processing unit. The two mirrors (M2 and M3) and two beam splitters
(BS1 and BS2) form the Mach-Zehnder interferometer, which includes acousto-optic modulators (AOM1 and AOM2), beam expanders (BE1 and BE2), and a focusing lens (L1), where the AOM1 and AOM2 are driven by $\Omega$ and $\Omega+\Delta \Omega$, respectively. In the Mach-Zehnder interferometer, the upper path beam becomes a spherical wave toward the object by BE1 and lens L1, and the lower path beam becomes a plane wave toward the object by BE2. The upper and lower path beams are interfered at BS2 and generate a so-called time-dependent Fresnel zone pattern (TD-FZP) onto the object, $I_{o}(x, y ; z)$, located $z$ away from the focal length of lens L1. The TD-FZP scans over the object through the use of the $x-y$ scanner, as shown in Fig. 1. The transmitted light, after collected by lens L2, is spatially integrated by photodetector PD1. The photodetector then converts the spatially integrated light to an electrical current that is the input to the electronic processing unit, which is composed of mixers and low-pass filters, as shown in Fig. 1. In the electronic processing unit, the in-phase and quadrature-phase components, $i_{r}(x, y ; z)$ and $i_{i}(x, y ; z)$ of the current are extracted. These in-phase and quadrature-phase signals are stored in a digital computer using an analog-to-digital converter. In the digital computer, the FP complex hologram of the object is synthesized by adding the in- and quadraturephase signals in the following manner [11]:

$$
\begin{aligned}
H_{\text {full }}(x, y) & =i_{r}(x, y ; z)-j i_{i}(x, y ; z)=\int_{z_{0}-\Delta z}^{z_{0}+\Delta z} I_{o}(x, y ; z) \\
& \otimes \frac{j}{\lambda z} \exp \left\{\left(\frac{-\pi}{\mathrm{NA}^{2} z^{2}}+j \frac{\pi}{\lambda z}\right)\left(x^{2}+y^{2}\right)\right\} \mathrm{d} z,
\end{aligned}
$$

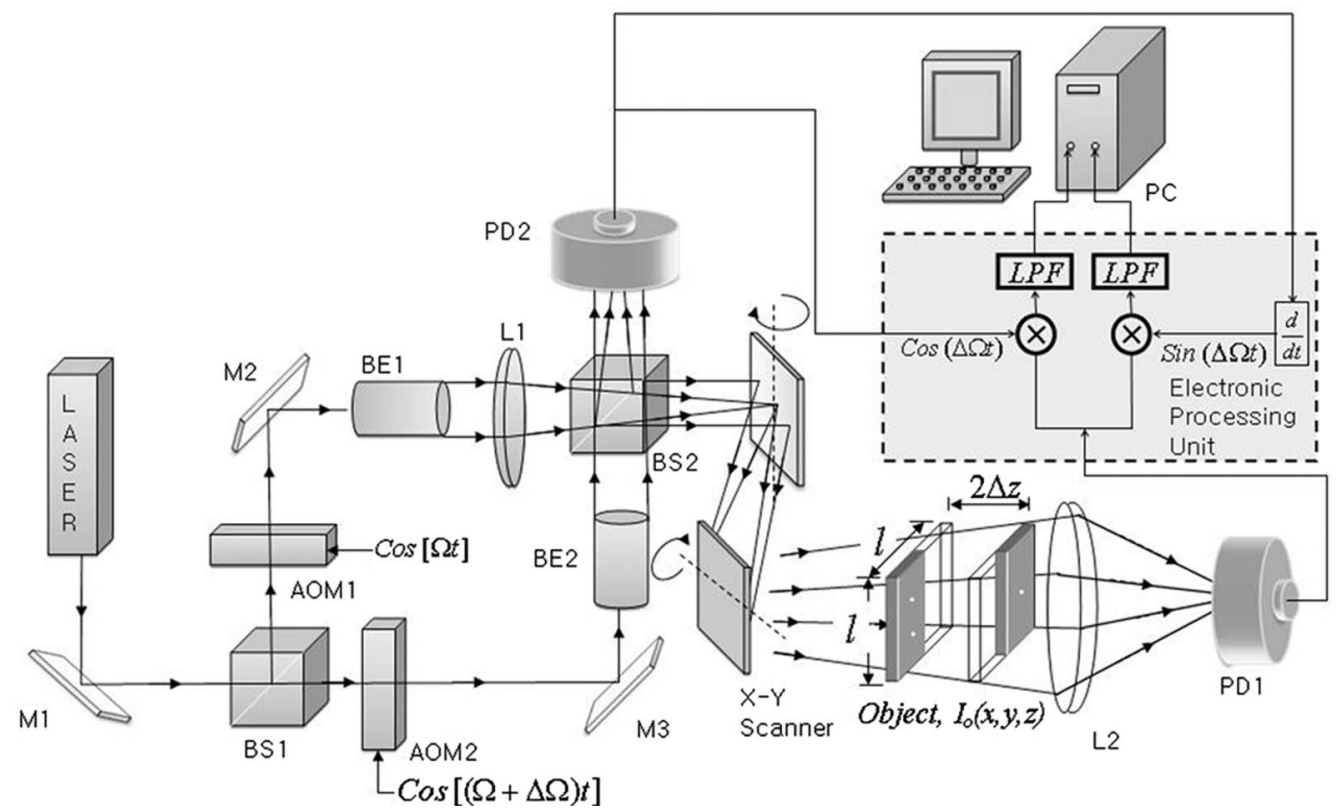

Fig. 1. Optical scanning holography (M, mirror; AOM, acousto-optic modulator; BS1,2, beam splitters; BE1,2, beam expanders; L, focusing lens; $\otimes$, electronic multiplexer; LPF, low-pass filter; PC, personal computer). 
where $z_{0}$ is the depth location of the object and $2 \Delta z$ is the depth range of the object, as shown in Fig. 1. The symbol $\otimes$ denotes the two-dimensional (2D) convolution operation defined as $g_{1}(x, y) \otimes g_{2}(x, y)=$ $\iint g_{1}\left(x /, y^{\prime}\right) g_{1}\left(x-x^{\prime}, y-y^{\prime}\right) \mathrm{d} x^{\prime} \mathrm{d} y^{\prime}$. Note that the extent of the FZP along the horizontal and vertical directions is determined by the numerical aperture (NA) of the Gaussian function shown in Eq. (1). Therefore, the recorded hologram has the same parallax along both the $x$ and $y$ directions. In addition, because the recorded hologram is encoded by the complex FZP, the hologram does not have a twin-image component. Accordingly, we call the recorded hologram an FP complex hologram. Because the recorded hologram is the encoded pattern between the object's intensity and complex FZP with an FP, the complex FP hologram can be considered as a collection of $2 \mathrm{D}$ complex FZPs [11]. Accordingly, the hologram can reconstruct the object's 3D image without twin-image noise with FP. However, a huge amount of data is required to represent FP information [12]. To reduce the required amount of data, an HPO hologram has been proposed for 3D display [1-5]. Recently, an algorithm that converts the FP hologram to an HPO hologram has also been proposed as an excellent way to reduce the required amount of data for 3D display [7]. While an FP hologram of a 3D object can be considered as a collection of 2D FZPs, an HPO hologram is a collection of 1D FZPs [7]. Accordingly, the HPO hologram can be considered as the encoding of the object's intensity with 1D FZPs, given as follows [7]:

$$
\begin{aligned}
H_{\mathrm{HPO}}(x, y) & =\int_{z_{0}-\Delta z}^{z_{0}+\Delta z} I_{0}(x, y ; z) \\
\otimes & \frac{j}{\lambda z} \exp \left[-\left(\frac{\pi}{\mathrm{NA}^{2} z^{2}}+j \frac{\pi}{\lambda z}\right) x^{2}\right. \\
& \left.-\frac{\pi}{\mathrm{NA}_{l p}^{2} z^{2}} y^{2}\right] \mathrm{d} z,
\end{aligned}
$$

where $\mathrm{NA}_{l p}$ determines the extent of the $1 \mathrm{D}$ FZP along the vertical direction. Here, we note that the 1D FZP has a limited extent without curvature along the vertical direction. In the following section, we review the conversion algorithm that converts the recorded complex FP hologram to the complex HPO hologram [7].

\section{HPO Hologram}

As discussed in Section 2, OSH can record the complex hologram of an object with FP as an electronic signal. In this section, we will review the algorithm that converts the complex FP hologram to an HPO hologram [7]. First, we filter the complex FP hologram to a complex HPO hologram using FM-GLF along the vertical direction:

$$
G_{\mathrm{FM}-\mathrm{GLF}}\left(k_{x}, k_{y}\right)=\exp \left[-\frac{1}{4 \pi}\left(\frac{\lambda}{\mathrm{NA}_{g}}\right)^{2} k_{y}^{2}\right] \exp \left(-j \frac{\lambda z_{0}}{4 \pi} k_{y}^{2}\right),
$$

where $\left(k_{x}, k_{y}\right)$ denotes spatial frequencies, $\mathrm{NA}_{g}$ is a parameter that determines the cutoff frequency of the FM-GLF, and $z_{0}$ is again the depth location of the object, which can be extracted directly from the complex FP hologram using a recently proposed depth detection technique [13]. The filtered hologram is then given by

$$
\begin{aligned}
H_{\mathrm{HPO}}(x, y)= & F^{-1}\left[F\left\{H_{\mathrm{full}}(x, y)\right\} G_{\mathrm{FM}-\mathrm{GLF}}\left(k_{x}, k_{y}\right)\right] \\
= & \int_{z_{o}-\Delta z}^{z_{o}+\Delta z} I_{o}(x, y, z) \\
& \otimes \frac{j}{\lambda z} \exp \left[-\left(\frac{\pi}{\mathrm{NA}^{2} z^{2}}+j \frac{\pi}{\lambda z}\right) x^{2}\right. \\
& \left.-\frac{\pi}{\mathrm{NA}_{l p}^{2} z^{2}} y^{2}\right] \mathrm{d} z
\end{aligned}
$$

where $F\{\bullet\}$ and $F^{-1}\{\bullet\}$ represent Fourier transformation and its inverse, and $\mathrm{NA}_{l p}=\mathrm{NA}_{g} \mathrm{NA} / \sqrt{\mathrm{NA}}$ $2+\mathrm{NA}_{g}^{2}$ is the numerical aperture of the FZP along the vertical direction, which determines the extent of the FZP along the vertical direction in the spatial domain. In FM-GLF, we adjust $\mathrm{NA}_{g}$ to make the infocus range of the FZP along the vertical direction, $2 \Delta z_{\text {ver_dir }}=2 \lambda / \mathrm{NA}_{l p}^{2}$, larger than the depth range of the object, $2 \Delta z$, which is usually true in case of synthesizing an HPO hologram for 3D display. Note that this is the exact expression of the HPO hologram of a computer-generated HPO hologram, as in Eq. (2). Here, the HPO hologram synthesized by FM-GLF becomes the encoded pattern between the object's intensity and the 1D FZP, whose extent along the vertical direction is determined by the cutoff frequency of the FM Gaussian low-pass filter. Figure 2 shows a flow chart of the proposed algorithm, and its first three blocks illustrate the procedures discussed so far. In the following discussion, we will investigate the data reduction rate between the HPO hologram and the FP hologram. Since NA determines the extent of the FZP that encodes the hologram, the extent of the 2D FZP is the same along the horizontal and vertical directions. When we represent the FP hologram on an SLM with the size of $l \times l$, according to the Nyquist sampling theorem, the required number of samples (or resolvable pixels) is given by $N_{\text {full-parallax }}=$ $(l / \Delta l)^{2}$. However, since the extent of 1D FZP depends on the lateral directions, the required pixel pitches of the HPO hologram along the horizontal and vertical directions are given by $\Delta l \leq \frac{\lambda}{2 \mathrm{NA}}$ and $\Delta l_{\mathrm{ver}} \leq \frac{\lambda}{2 \mathrm{NA}_{l}}$, respectively. Thus, when we represent the HPO hologram on an SLM with the size of $l \times l$, the required number of samples is given by $N_{\mathrm{HPO}}=(l / \Delta l) \times$ $\left(l / \Delta l_{\text {ver }}\right)$. The ratio of the required numbers of samples between the HPO hologram and the FP hologram is defined as a data reduction ratio and given by [7]: 


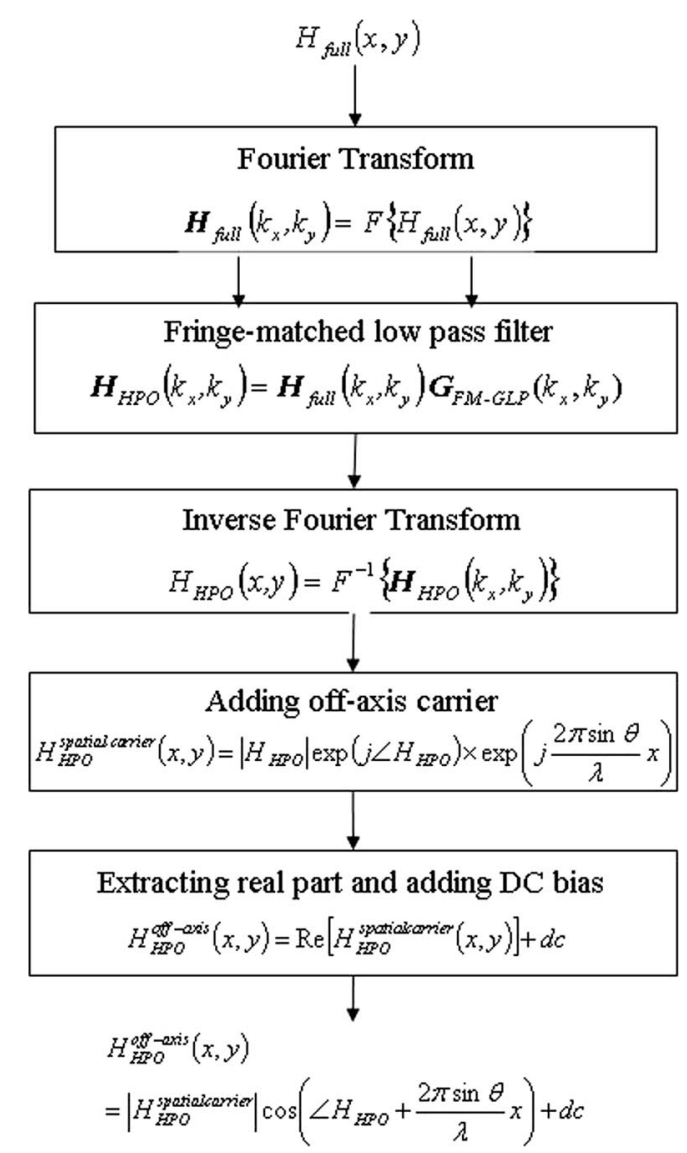

Fig. 2. Flow chart for off-axis HPO real hologram conversion.

$$
R=\frac{N_{\mathrm{HPO}}}{N_{\mathrm{Full}}}=\frac{\mathrm{NA}_{l p}}{\mathrm{NA}}
$$

\section{Conversion to an Off-Axis HPO Hologram and Reconstruction with Amplitude-Only SLM}

We have converted a complex FP hologram to a complex HPO hologram, as discussed in Section 3. Because a complex hologram records the amplitude and the complete phase of the diffracted optical field, reconstruction of the complex hologram can give the $3 \mathrm{D}$ image of an object without the twin-image and background noise [14]. However, a conventional SLM cannot represent a complex HPO hologram because the SLM can represent either amplitude or phase. Because a conventional amplitude-only SLM can modulate the amplitude of an optical field, we can represent either the real or the imaginary part of the complex HPO hologram with DC bias. However, when we reconstruct the 3D image of the object optically for 3D display using an amplitude-only SLM, the reconstructed 3D image of the object is corrupted by the twin-image and background noise. On the other hand, a phase-only SLM modulates only the phase of an optical field, and, thus, if we represent the phase of the complex HPO hologram using the phase-only SLM, the reconstructed 3D image is distorted by amplitude flattening. In this section, we will propose further to convert the complex HPO ho- logram to an off-axis HPO hologram that is composed of real value only but can reconstruct the $3 \mathrm{D}$ image of an object without the twin-image noise, background noise, and the distortion by amplitude flattening. After conversion, we optically reconstruct an off-axis HPO hologram using a conventional amplitude-only SLM for 3D display. In the off-axis hologram, the optical axis of the reference wave is tilted to that of the object wave. The angle between the optical axes of the reference and object waves introduces a spatial carrier within the hologram. The spatial carrier allows the separation of the desired 3D image of the object from the twin-image noise and background noise in the reconstruction stage $[15,16]$. To convert the complex HPO hologram to an off-axis HPO hologram, we multiply a spatial carrier term to Eq. (3):

$$
\begin{aligned}
& H_{\mathrm{HPO}}^{\text {spatial carrier }}(x, y)=\left|H_{\mathrm{HPO}}\right| \exp \left(j \angle H_{\mathrm{HPO}}\right) \\
& \times \exp \left(j \frac{2 \pi \sin \theta}{\lambda} x\right) \\
& =|H| \exp \left[j\left(\angle H_{\mathrm{HPO}}+\frac{2 \pi \sin \theta}{\lambda} x\right)\right] \text {, }
\end{aligned}
$$

where $\angle$ represents the phase of a complex function, and $\theta$ is a tilted angle between the optical axes of the reference and object waves. Note that we need to choose the off-axis angle, $\theta$, large enough to separate the desired 3D image from the twin-image and background noise $[14,15]$. The spatial carrier that is added to the complex HPO hologram separates the background noise as the zeroth-order beam and the twin image as the first-order beam, which are spatially separated from the desired 3D image in the optical reconstruction. [14,15]. To acquire an off-axis HPO real hologram suitable for display on an amplitude-only SLM, we extract the real part of Eq. (ㅁ) and add a DC bias to give

$$
\begin{aligned}
H_{\mathrm{HPO}}^{\text {off-axis }}(x, y)= & \operatorname{Re}\left[H_{\mathrm{HPO}}^{\text {spatial carrier }}(x, y)\right]+d c \\
= & \left|H_{\mathrm{HPO}}^{\text {spatial carrier }}\right| \cos \left(\angle H_{\mathrm{HPO}}\right. \\
& \left.+\frac{2 \pi \sin \theta}{\lambda} x\right)+d c,
\end{aligned}
$$

where $d c$ is a DC bias added to make the off-axis HPO hologram become a positive value. Figure 2 shows the flow chart of the proposed algorithm; its last two blocks illustrate the off-axis conversion procedures discussed so far. From the off-axis real HPO hologram given in Eq. (5), we can optically reconstruct the 3D image of an object using a conventional amplitude-only hologram with data reduction, as shown in Fig. 3. The optical reconstruction system consists of a laser, a spatial filter beam (SP), a polarizer (PL), a beam splitter (BS), an amplitudeonly SLM, and an analyzer (AL). First, we load 


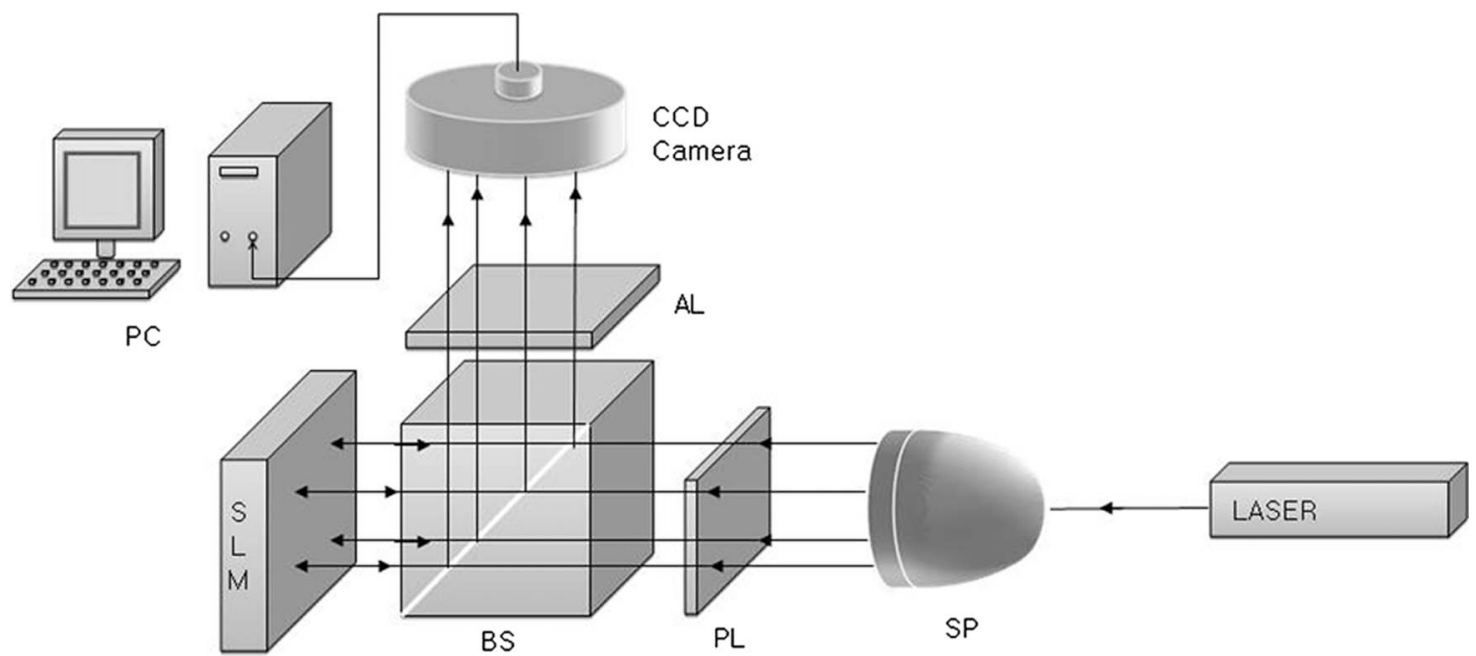

Fig. 3. Reconstruction stage with amplitude-only SLM (PC, personal computer; AL, analyzer; SLM, spatial light modulator; BS, beam splitter; PL, polarizer; SP, spatial filter).

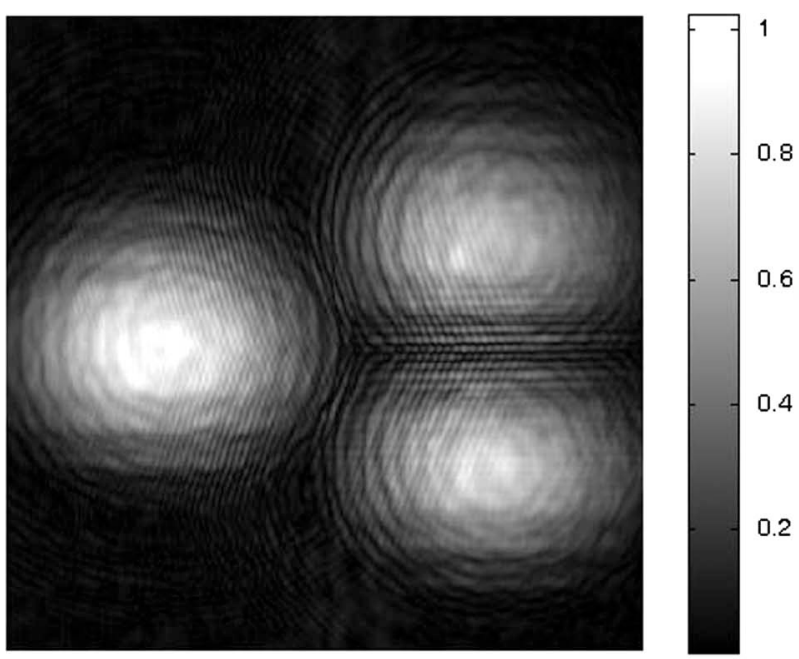

(a)

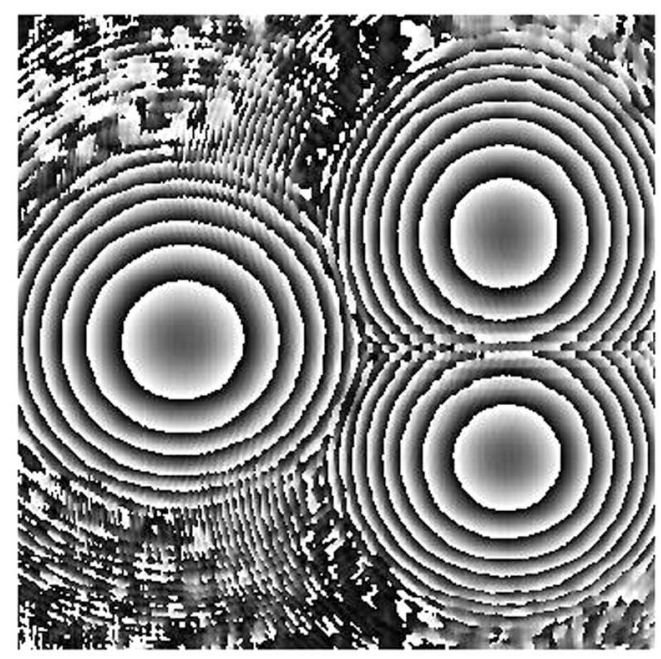

(b)

the digitally converted off-axis HPO real hologram [see Eq. (6)] to the amplitude-only SLM. The collimated laser beam is polarized by PL, which is then illuminated onto the SLM through the BS. The polarization state of the illuminated light is modulated spatially according to the loaded off-axis HPO hologram. The spatially modulated light is reflected from the SLM and the BS and finally analyzed by the AL. The AL changes the modulated polarization state into amplitude distribution, which reconstructs the $3 \mathrm{D}$ image of the object. The CCD camera then records the reconstructed desired 3D image.

\section{Experimental Results}

OSH is used to record the complex FP hologram of a real object composed of three points on two slides, as shown in Fig. 1. Two points are located on the front slide, and one point is located on the back slide. The diameter of the points is $200 \mu \mathrm{m}$ and the depth distance between the two slides is $2 \Delta z=20 \mathrm{~cm}$. In the front slide, the two points are separated by $4 \mathrm{~mm}$. The
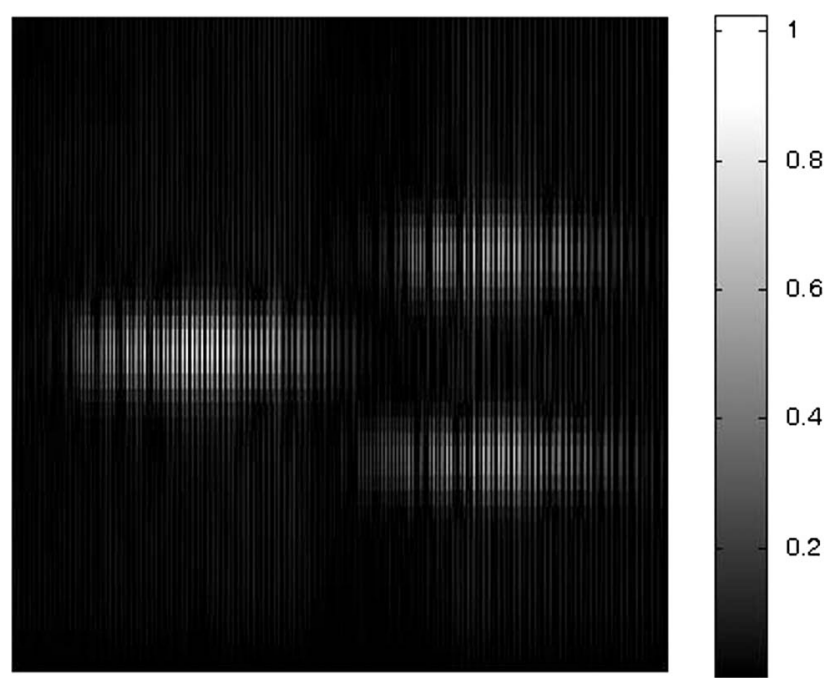

Fig. 5. Off-axis HPO real hologram. 
front slide of the object is located at $77 \mathrm{~cm}$ away from BS2, as shown in Fig. 1 . The wavelength of the laser output is $\lambda=633 \mathrm{~nm}$ and the AOM1,2 are driven by 40 and $40.01 \mathrm{MHz}$, respectively. The diameter of the collimated beam is $D=25 \mathrm{~mm}$ and the focal length of $\mathrm{L} 1$ is $f=500 \mathrm{~mm}$. Thus, the NA of the recorded complex FP hologram becomes $N A \approx D /(2 f)=0.025$. Scanning is performed along the lateral direction over an area of $1.4 \mathrm{~cm} \times 1.4 \mathrm{~cm}$ as a raster pattern. Figures 4(a) and 4(b) are the amplitude and phase parts of the complex FP hologram of the object [see Eq. (1)]. According to the first three parts of the flow chart shown in Fig. 2, we convert the FP hologram to an HPO hologram in which we set the filter parameter $\mathrm{NA}_{g}=0.00056$. This reduces the NA along the vertical direction to $\mathrm{NA}_{l p}=0.00056$, which gives the data reduction ratio of $R=\mathrm{NA}_{l p} / \mathrm{NA}=$ 0.022 according to Eq. (4). After conversion to the complex HPO hologram, we convert the hologram to an off-axis HPO real hologram according to the

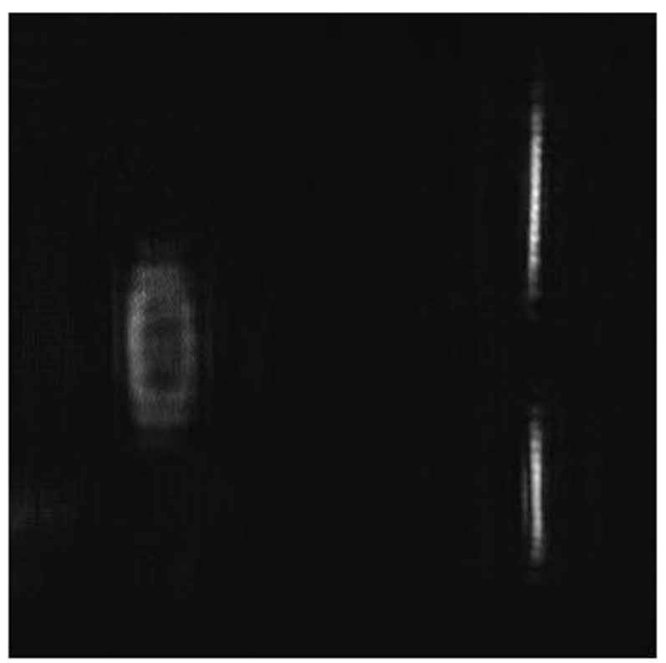

(a)

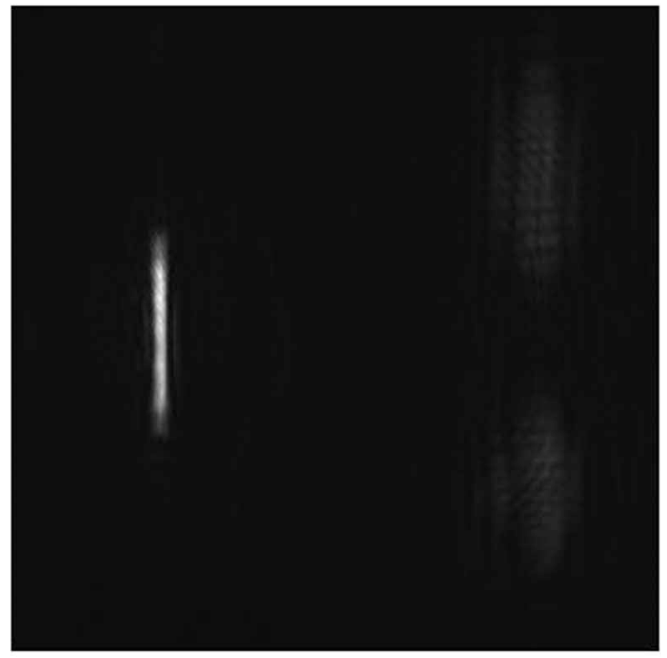

(b)

Fig. 6. (a) Reconstructed image at the location of the front slide with the off-axis real HPO hologram; (b) reconstructed image at the location of the back slide with the off-axis real HPO hologram. last two parts of the block diagram shown in Fig. 2. The converted off-axis HPO real hologram is shown in Fig. 5. In the reconstruction stage, we load the offaxis $\mathrm{HP} O$ real hologram onto the SLM, which is an LC-R 2500 from HoloEye. as shown in Fig. 3. The size and the resolution of the SLM are $19.5 \mathrm{~mm} \times$ $14.6 \mathrm{~mm}$ and $1024 \times 768$, respectively with a pixel pitch of $19 \mu \mathrm{m}$. The reconstructed 3D image of the object is recorded with a CCD camera. The reconstructed images at the location of the front and back slides are shown in Figs. $\underline{6(\mathrm{a})}$ and $\underline{6(\mathrm{~b})}$. Note that we can see that the reconstructed images are focused at each corresponding depth location without the twinimage and background noises. In order to see the effect of the twin-image and background noise, we reconstruct the real part of the complex HPO hologram with DC bias that is the on-axis hologram of the recorded object. Figures 7(a) and 7(b) show the reconstructed images at the location of the front and back

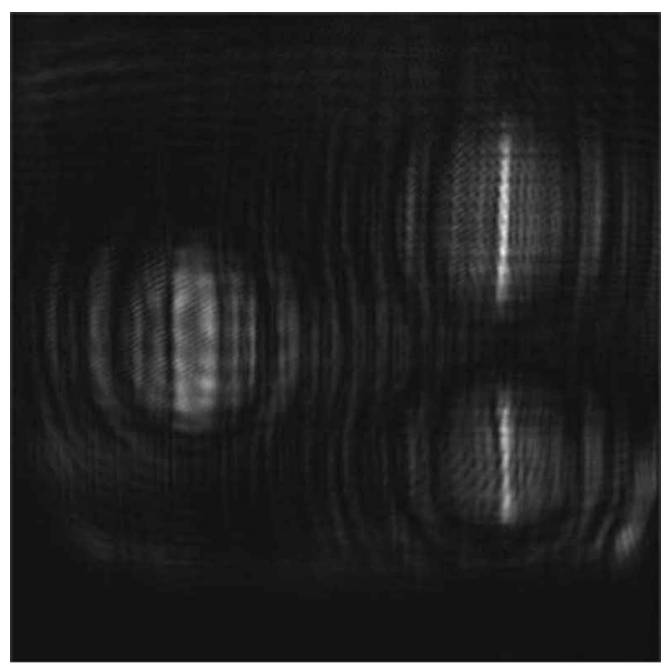

(a)

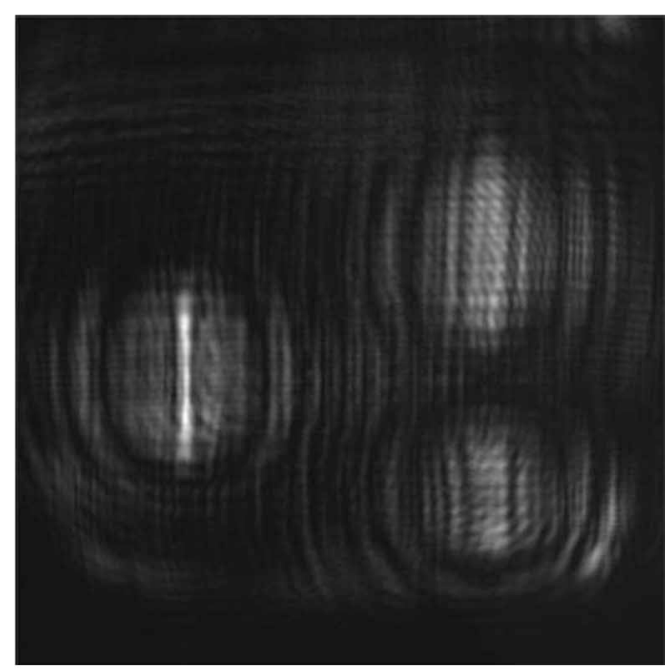

(b)

Fig. 7. (a) Reconstructed image at the location of the front slide with the on-axis HPO hologram; (b) reconstructed image at the location of the back slide with the on-axis HPO hologram. 
slides. We can see that the reconstructed images are corrupted by twin-image and background noises.

\section{Conclusion}

We have proposed to reconstruct an optically recorded HPO hologram by converting a complex FP hologram into an off-axis HPO hologram for 3D display. First, we record the complex FP hologram using OSH. Second, we digitally convert the complex FP hologram to an off-axis HPO hologram using FMGLF and the addition of an off-axis spatial carrier. Finally, we reconstruct the off-axis HPO hologram using an amplitude-only SLM. To the best of our knowledge, this is the first demonstration that records and displays an HPO hologram of an object optically. Only computer-generated HPO holograms, hitherto, have been displayed. The technique proposed here is not limited to holograms recorded by $\mathrm{OSH}$, but is also applicable to any complex FP hologram obtained by a conventional holographic technique, such as phase-shifting holography.

This research was supported by Basic Science Research Program through the National Research Foundation of Korea (NRF) funded by the Ministry of Education, Science and Technology (20090087476).

\section{References}

1. P. S. Hilaire, S. A. Benton, and M. Lucente, "Synthetic aperture holography: a novel approach to three-dimensional displays," J. Opt. Soc. Am. A 9, 1969-1977 (1992).

2. T. Yamaguchi, G. Okabe, and H. Yoshikawa, "Real-time image plane full-color and full-parallax holographic video display system," Opt. Eng. 46, 125801 (2007).

3. T. Ito, T. Shimobaba, H. Godo, and M. Horiuchi, "Holographic reconstruction with a $10 \mu \mathrm{m}$ pixel-pitch reflective liquid- crystal display by use of a light-emitting diode reference light," Opt. Lett. 27, 1406-1408 (2002).

4. M. Stanley, M. A. Smith, A. P. Smith, P. J. Watson, S. D. Coomber, C. D. Cameron, C. W. Slinger, and A. D. Wood, "3D electronic holography display system using a 100-megapixel spatial light modulator," Proc. SPIE 5249, 297-308 (2004).

5. Lucente, "Diffraction-specific fringe computation for electroholography," Ph.D. dissertation, Program in Electrical Engineering and Computer Science (Massachusetts Institute of Technology 1994).

6. T.-C. Poon, T. Akin, G. Indebetouw, and T. Kim, "Horizontalparallax-only electronic holography," Opt. Express 13 , 2427-2432 (2005).

7. T. Kim, Y. S. Kim, W. S. Kim, and T.-C. Poon, "Algorithm for converting full-parallax holograms to horizontalparallax-only holograms," Opt. Lett. 34, 1231-1233 (2009).

8. N. T. Shaked and J. Rosen, "Modified Fresnel computergenerated hologram directly recorded by multiple-viewpoint projections," Appl. Opt. 47, D21-D27 (2008).

9. T.-C. Poon, "Scanning holography and two-dimensional image processing by acousto-optic two-pupil synthesis," J. Opt. Soc. Am. A 2, 521-527 (1985).

10. T.-C. Poon, "Optical scanning holography—a review of recent progress," J. Opt. Soc. Korea 13, 406-415 (2009).

11. T.-C. Poon, "On the fundamentals of optical scanning holography,” Am. J. Phys. 76, 738-745 (2008).

12. T.-C. Poon, "Three-dimensional television using optical scanning holography," J. Inf. Display 3, 12-16 (2002).

13. T. Kim and T.-C. Poon, "Autofocusing in optical scanning holography," Appl. Opt. 48, H153-H159 (2009).

14. T.-C. Poon, T. Kim, G. Indebetouw, B. W. Schilling, M. H. Wu, K. Shinoda, and Y. Suzuki, "Twin-image elimination experiments for three-dimensional images in optical scanning holography," Opt. Lett. 25, 215-217 (2000).

15. E. N. Leith and J. Upatnieks, "Reconstructed wavefronts and communication theory," J. Opt. Soc. Am. 52, 1123-1130 (1962).

16. E. N. Leith and J. Upatnieks, "Wavefront reconstruction with continuous-tone objects,” J. Opt. Soc. Am. 53, 1377-1381 (1963). 